\title{
Long-term suppression of telomerase expression in HeLa cell clones, transfected with an expression vector carrying siRNA targeting hTERT mRNA
}

\author{
KAISA KURVINEN $^{1}$, STINA SYRJÄNEN ${ }^{1}$ and BO JOHANSSON ${ }^{2}$ \\ ${ }^{1}$ Department of Oral Pathology and Oral Radiology, Institute of Dentistry and MediCity Research Laboratory, \\ Faculty of Medicine, University of Turku, Turku, Finland; ${ }^{2}$ Department of Clinical Microbiology, \\ Karolinska Institutet, Karolinska University Hospital, Stockholm, Sweden
}

Received November 25, 2005; Accepted January 16, 2006

\begin{abstract}
HeLa cell cultures were used as model systems for small interfering RNA (siRNA) induced knockdown of mRNA expression of the human telomerase catalytic subunit, telomerase reverse transcriptase (hTERT). Four 21-bp siRNAs targeting different sites of the hTERT mRNA were designed, and the siRNA molecules produced by a T7 transcription system in vitro. In transient transfection assays on HeLa cells, only one of the tested siRNAs produced a potent knockdown effect on hTERT mRNA expression, associated with the suppression of telomerase activity (both reduced by $\sim 50 \%$ ). An expression vector encoding a hairpin siRNA against the effective hTERT mRNA target site was generated, and HeLa clones stably expressing hTERT-specific siRNA were created. Two clones showed extremely reduced hTERT mRNA expression, associated with unusually short telomeres, the inhibition of cell growth and the induction of senescence and apoptosis. Thus, there was obvious loss of viability in cells lacking hTERT expression and carrying short telomeres. This was most prominent in the clone that showed prolonged reductions (over two months) in both hTERT expression and telomerase activity. Thus, our data clearly show that long-term suppression of telomerase expression by siRNA is an attainable goal, at least in a HeLa cell model system.
\end{abstract}

\section{Introduction}

Telomeres are specialized DNA-protein structures that cap the ends of chromosomes. These telomeric structures protect chromosome ends from degradation and end-to-end fusions

Correspondence to: Professor Stina Syrjänen, Department of Oral Pathology and Oral Radiology, Institute of Dentistry, Faculty of Medicine, University of Turku, Lemminkäisenkatu 2, 20520 Turku, Finland

E-mail: stina.syrjanen@utu.fi

Key words: telomerase, hTERT, mRNA, siRNA and therefore are crucial for maintaining genomic integrity and stability $(1,2)$. In most human somatic cells, telomeres shorten gradually with each cell division due to the endreplication problem in linear DNA replication $(1,3)$. When telomeres reach a critical length, cells stop dividing and enter into replicative senescence. Cells that bypass this senescent state eventually undergo telomere crisis, accompanied by chromosomal instability and increased cell death $(1,4)$.

For maintaining proliferative potential, cells need to prevent the progressive shortening of telomeres. This is most frequently achieved by the activation of telomerase. Telomerase is a ribonucleoprotein enzyme that compensates telomere erosion by adding telomeric DNA repeats to chromosome ends $(1,2,4)$. Two main components, an RNA subunit (hTR) and a catalytic subunit, telomerase reverse transcriptase (hTERT) form the telomerase active site $(2,4)$. While the hTR component is constitutively expressed in all cell types, the expression of hTERT is tightly regulated, indicating that hTERT is a rate-limiting factor for the enzyme $(4,5)$. In contrast to most normal somatic cells with repressed telomerase activity, telomerase is transiently induced in certain stem or progenitor cells, and constitutively expressed by germ cells and most cancer cells (4-7). About $90 \%$ of all human tumor cells exhibit high levels of telomerase activity through the transcriptional upregulation of hTERT $(5,6)$. Accordingly, telomerase appears to have a key role in contributing to the immortality of cancer cells.

Studies using dominant-negative mutants of hTERT protein have provided direct evidence that telomerase activity is essential for the maintenance of long-term viability of tumor cells. It was shown that the introduction of hTERT mutants into telomerase-positive human cancer cells inhibits telomerase activity, causing telomere shortening and eventually inducing cell growth inhibition and apoptosis $(8,9)$. These observations strongly support that telomerase is a promising target for inhibiting tumor cell proliferation and that targeting the catalytic subunit of the enzyme offers one potential approach for diminishing telomerase function. Several successful strategies have been described to inhibit telomerase activity by targeting hTERT, including the use of antisense oligonucleotides, ribozymes and inhibitory agents (10-12). RNA interference (RNAi) has proven to be a powerful tool for blocking the 
expression of target genes specifically, thus holding obvious therapeutic potential (13). This technology has also been utilized to knockdown the expression of hTERT mRNA in cancer cells. Short-term experiments have shown that small interfering RNAs (siRNAs) can induce potent inhibitory effects on hTERT expression, resulting in decreased telomerase activity and cell growth properties (14-16).

Studies of the highly telomerase-positive cervical carcinoma cell line HeLa have demonstrated that a mass cell population with a stable mean telomere length contains subclones with large differences in telomere length and telomerase activity (17). In this study, to shed light on the telomerase-dependent telomere length regulation for better understanding of inhibitory effects on this process, we first isolated subclones of the HeLa cell line and evaluated correlations between the levels of hTERT mRNA expression and telomerase activity and their role in the regulation of telomere length. In order to conduct long-term gene silencing of hTERT in cancer cells, we applied a DNA vector-based system to express constitutively siRNA against hTERT in the cells. We created stable transfected HeLa clones and explored the long-term effects of potent hTERT suppression on telomerase activity, telomere length and viability of the cells.

\section{Materials and methods}

Cell cultivation. The human cervical carcinoma cell line HeLa was grown in Dulbecco's modified Eagle's medium (Gibco BRL Life Technologies, Gaithersburg, MD, USA) supplemented with $10 \%$ fetal bovine serum (Gibco BRL Life Technologies) at $37^{\circ} \mathrm{C}$ and $5 \% \mathrm{CO}_{2}$ in a humidified atmosphere. Cells were regularly subcultured to maintain exponential growth. HeLa subclones were isolated by a limiting dilution method. For transfection, HeLa cells were seeded at the density of $10 \times 10^{3}$ cells $/ \mathrm{cm}^{2}$, and the cells were allowed to grow in antibiotic-free complete growth media for $24 \mathrm{~h}$ before transfection. The cells were about $40-50 \%$ confluent at the time of transfection.

Analysis of hTERT mRNA expression. hTERT mRNA levels were measured by real-time RT-PCR. Total RNA was isolated from the cells by TRIzol Reagent (Gibco BRL Life Technologies) using the manufacturer's single-step chloroformextraction protocol. Complementary DNA was synthesized using the First-strand cDNA synthesis kit by Amersham Biosciences (Piscataway, NJ, USA). An aliquot of $1 \mu \mathrm{g}$ of total RNA was reverse transcripted by MMLV transcriptase using random hexamer primers according to the manufacturer's instructions. Real-time RT-PCR was performed using cDNA corresponding to $200 \mathrm{ng}$ of total RNA in a $25 \mu \mathrm{l}$ PCR reaction and using the TaqMan Universal Master Mix (Applied Biosystems, Foster City, CA, USA) and primers and probe developed by Applied Biosystems. Quantifications were performed in triplicates using the ABI PRISM 7700 Sequence Detection System (Applied Biosystems) with amplification conditions: $2 \mathrm{~min}$ at $50^{\circ} \mathrm{C}, 10 \mathrm{~min}$ at $95^{\circ} \mathrm{C}$, a two-step cycle at $95^{\circ} \mathrm{C}$ for $15 \mathrm{sec}$ and $60^{\circ} \mathrm{C}$ for $60 \mathrm{sec}$ for a total of 40 cycles. Ribosomal RNA (rRNA) abundance was measured in all samples as an endogenous control using an aliquot of cDNA corresponding to $1 \mathrm{ng}$ of RNA in a $25 \mu 1 \mathrm{PCR}$ reaction and using primers and probe provided by Applied Biosystems. The raw abundance values for hTERT mRNA were normalized against the raw abundance values of rRNA.

Analysis of telomerase activity. Telomerase activity was measured using a recently described SYBR Green real-time telomeric repeat amplification protocol (TRAP) (18). Samples for telomerase activity assays were extracted following a standard protocol. Briefly, cell samples containing 100000 cells were transferred to Eppendorf tubes. The cell pellets were washed twice with cold phosphate-buffered saline (PBS) and then resuspended in ice-cold TRAP lysis buffer (1000 cells $/ \mu \mathrm{l})$ and incubated for $30 \mathrm{~min}$ on ice. After centrifugation, aliquots of the supernatant were rapidly frozen and stored at $-70^{\circ} \mathrm{C}$. Quantification of telomerase activity was performed with an aliquot of cell extract corresponding to 1000 cells in a $25 \mu 1$ PCR reaction containing SYBR Green PCR Master Mix (Applied Biosystems) and telomerase primers at concentrations described in the previously published protocol (18). All measurements were performed in triplicates using the GeneAmp 5700 Sequence Detection System (Applied Biosystems) with amplification conditions: $20 \mathrm{~min}$ at $25^{\circ} \mathrm{C}, 10 \mathrm{~min}$ at $95^{\circ} \mathrm{C}$, a two-step cycle at $95^{\circ} \mathrm{C}$ for $30 \mathrm{sec}$ and $60^{\circ} \mathrm{C}$ for $90 \mathrm{sec}$ for a total of 35 cycles. Standard curves consisted of HeLa cells diluted with lysis buffer to final cell numbers of 1,10 , 100 and 1000. Protein concentrations of cell extracts was determined by Bradford assay (Bio-Rad Laboratories, Hercules, CA, USA). Telomerase activity results were normalized against protein concentrations.

Telomere length analysis. Telomere length analysis was performed by the Southern blot analysis of telomeric restriction fragments (TRFs) according to the protocol kindly provided by Dr Renske Steenbergen (VU University Medical Center, Amsterdam, the Netherlands). Genomic DNA was extracted from cells according to the method of Miller and colleagues (19). A $2.5 \mu \mathrm{g}$ sample of DNA was digested with HinfI and RsaI (New England Biolabs, Beverly, MA, USA), separated in a $0.7 \%$ agarose gel at $30 \mathrm{~V}$ for $18 \mathrm{~h}$ and transferred onto a nylon membrane. The membrane was hybridized with telomeric oligonucleotide probe (TTAGGG) ${ }_{4}$ end-labeled with $\left[\gamma^{-32} \mathrm{P}\right] \mathrm{ATP}$ (Amersham Biosciences) and then exposed to a phosphor-imager screen. The screen was scanned using the BAS 5000 Image Analysis System (Fujifilm Medical Systems, Stamford, CT, USA). Telomere length (i.e., the size distribution of the TRFs) was estimated in comparison to a DNA length standard.

Morphology. For cytological analysis using light microscope, cells grown on cell culture coverslips were washed twice with PBS, fixed in $4 \%$ paraformaldehyde and stained with hematoxylin and eosin (HE).

Selection of siRNA sequences targeting hTERT mRNA. Four 21-mer siRNA sequences directed against hTERT mRNA were designed by a random selection method as described previously (20). All siRNA sequences were analyzed using a BLAST-search in the GenBank database, to confirm that only hTERT mRNA was targeted. siRNA sequences used in the study (in DNA oligonucleotide form) and their target 
sites on hTERT mRNA were as follows: siRNA-1, 5'AAGCACTTCCTCTACTCCTCA-3' (985-1005); siRNA-2, 5'-AATCAGACAGCACTTGAAGAG-3' (1764-1784); siRNA-3, 5'-AAGAGCCACGTCTCTACCTTG-3' (22782298); siRNA-4, 5'-AACATCTACAAGATCCTCCTG-3' (2809-2829).

Transfection assays with in vitro synthesized siRNAs. siRNAs were prepared by in vitro transcription using the Silencer ${ }^{\mathrm{TM}}$ siRNA Construction Kit according to the manufacturer's protocol (Ambion, Austin, TX, USA). The day before transfection, HeLa cells were seeded onto a 24-well plate at a density of $20 \times 10^{3}$ cells/well and allowed to grow for $24 \mathrm{~h}$. The cells were transfected with 20-50 nM concentrations of siRNAs complexed with GeneEraser ${ }^{\mathrm{TM}}$ siRNA transfection reagent (Stratagene, La Jolla, CA, USA) according to the manufacturer's instructions. Forty-eight hours after transfection, the cells were harvested for further analysis. As a control in the transfection experiments was used the cells transfected with the scrambled siRNA with no significant homology to any known human gene sequence. Oligonucleotide templates for in vitro synthesis of the scrambled siRNA were available from Ambion.

Construction of hTERT-siRNA expression vector. After the most effective siRNA was identified, a hairpin siRNA insert encoding siRNA for that effective hTERT target site was cloned in an siRNA expression vector under the control of the H1 RNA pol III promoter (pSilencer ${ }^{\mathrm{TM}}$ 3.1-H1 neo Expression Vector, Ambion) according to the manufacturer's instructions. To verify a construct of the hTERT-siRNA expression vector, the hairpin siRNA insert was sequenced. The siRNA expression vector used contained a neomycin resistance gene for enabling G418 selection of transfected cells.

Transient transfection with siRNA expression vector. Twentyfour hours before transfection, HeLa cells were plated onto a 24-well plate at a density of $20 \times 10^{3}$ cells/well. siPORT ${ }^{\mathrm{TM}}$ XP-1 transfection agent (Ambion) was used to deliver DNA plasmids into cells according to the manufacturer's protocol. A 3:1 or 6:1 ratio of transfection agent $(\mu 1)$ to plasmid DNA $(\mu \mathrm{g})$ was used for the formation of siPORT XP-1/DNA complexes, which were added to the growing cells in normal growth medium. A vector that expressed a scrambled hairpin siRNA with no significant homology to any known human gene sequences (pSilencer neo Negative Control Vector, Ambion) was used as a control. In transient transfection assays, the cells were harvested 48 or $72 \mathrm{~h}$ after transfection for further analysis.

Generation of stable clones of HeLa cells expressing hTERTsiRNA. HeLa cells were transfected with the hTERT-siRNA expression vector by the procedure described above. Twentyfour hours after transfection, G418 selection was started. The cells were cultured in normal growth medium containing $400 \mu \mathrm{g} / \mathrm{ml}$ G418 (Calbiochem, EMD Biosciences, San Diego, CA, USA) for 5 days, i.e., until all of the non-transfected cells were killed. After stable selection, the cells were plated at low density to grow into colonies. Single-cell clones were then removed with a cloning ring to grow in a 96-well culture plate. After clonal expansion, the hTERT-siRNA expressing cells were cultured under $250 \mu \mathrm{g} / \mathrm{ml} \mathrm{G} 418$ to prevent the accumulation of cells that had lost the expression vector. Cell populations were passed every 3-4 days, and cell samples were collected for different analyses during passaging, i.e., passage 3, 6, 8, 10 and 16 were corresponding to 30, 40, 47, 54 and 74 days after transfection, respectively. Accordingly, number of passages over 20 correspond to times over three months after transfection. Control HeLa cell populations were stably transfected with the control siRNA vector expressing a scrambled hairpin siRNA.

\section{Results}

Telomerase expression and telomere length in HeLa mass culture. In the present study, the levels of hTERT mRNA expression and telomerase activity were quantitatively assessed by using real-time RT-PCR and SYBR Green real-time telomeric repeat amplification protocol (TRAP) assay, respectively. Telomere length was determined by measuring the mean length of telomeric restriction fragments (TRFs) of genomic DNA with a Southern blot analysis. HeLa mass culture did not exhibit significant fluctuations in its hTERT mRNA expression or telomerase activity levels during culture (data not shown). The mean telomere length of HeLa cell population was about $5 \mathrm{~kb}$ (Fig. 2), and its telomere length remained unchanged during passaging (data not shown).

hTERT mRNA expression and telomerase activity in HeLa subclones. The levels of hTERT mRNA and telomerase activity were analyzed in $16 \mathrm{HeLa}$ subclones during passaging. All HeLa subclones were analyzed at least at two time-points. The first samples were collected three weeks after cloning, corresponding to passages 3-4 of the subclones, and the next samples were collected about two weeks later, corresponding to passages 6-9. Additionally, five of the subclones were analyzed about eight weeks after cloning, corresponding to passages 14-16 (Fig. 1). Fig. 1 demonstrates that HeLa subclones exhibited great variations in their hTERT mRNA expression and telomerase activity levels. At early passages (passage 3/4), individual clones showed a $\sim 40$-fold variation in hTERT mRNA expression level, the relative expression levels ranging from 4 to $155 \%$ compared to the level of hTERT mRNA expression in HeLa cell population (Fig. 1A). Similarly, telomerase activity levels were highly variable between individual clones, ranging from 23 to $142 \%$ of the telomerase activity of HeLa cells (Fig. 1B). Telomerase activity levels were generally proportionally higher than hTERT mRNA levels (in 12 of the 16 subclones), but also this ratio appeared to be highly variable (range from 1.2 to 12.5 -fold at early passages, and from 1.2 to 7.1 -fold in later passages). An overall observation of the subclones reveals that telomerase activity especially increased in all of the subclones initially with lower levels (Fig. 1B; Sc 7, 8 and 12), and all the subclones remained or regained $\geq 50 \%$ telomerase activity over time.

Telomere lengths of HeLa subclones. The HeLa subclones showed great variations in their telomere lengths, a range of mean TRF length being from about 2.0 to $8.0 \mathrm{~kb}$ (Fig. 2). The shortest telomere lengths with a range of 2.0 to $2.3 \mathrm{~kb}$ 
A

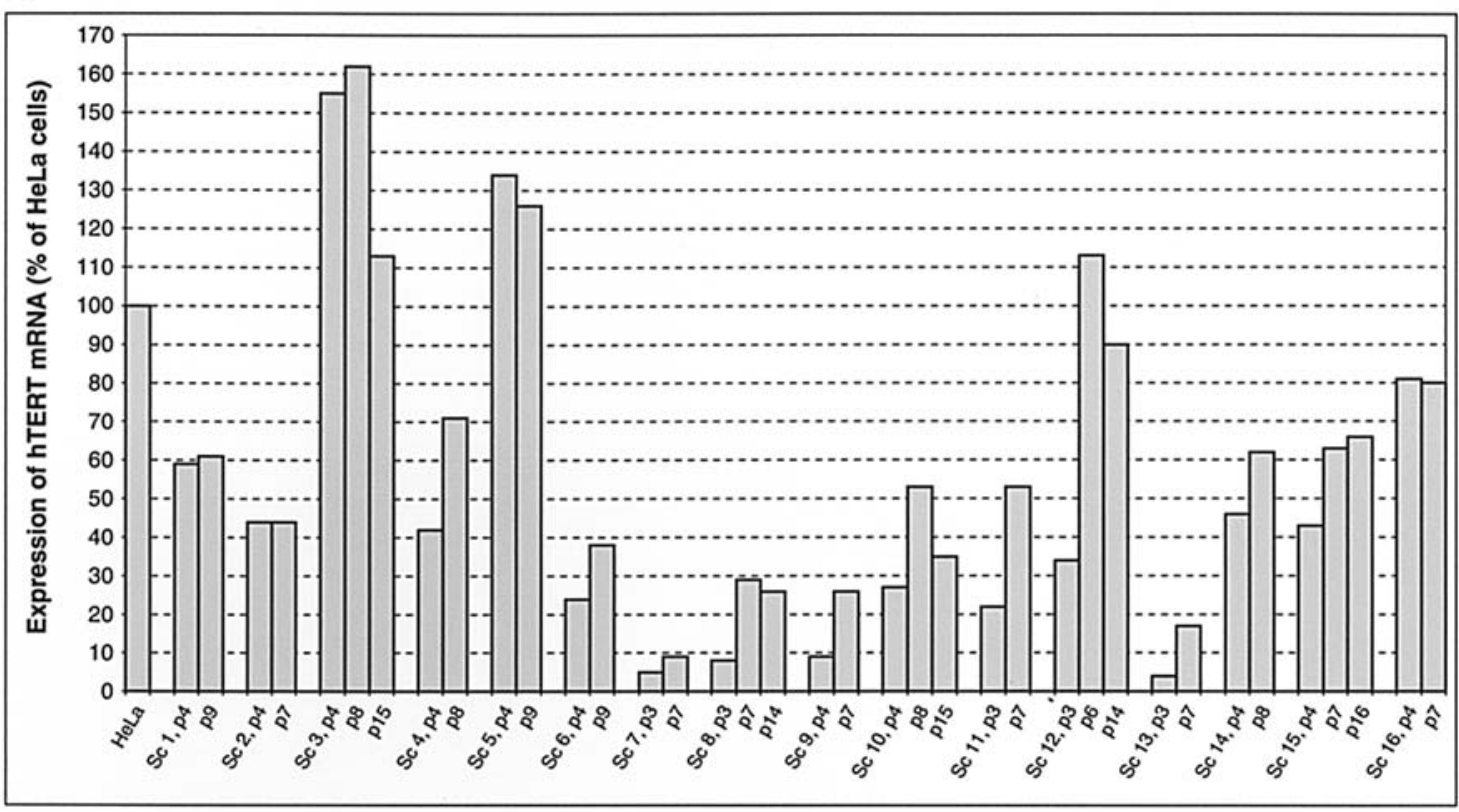

B

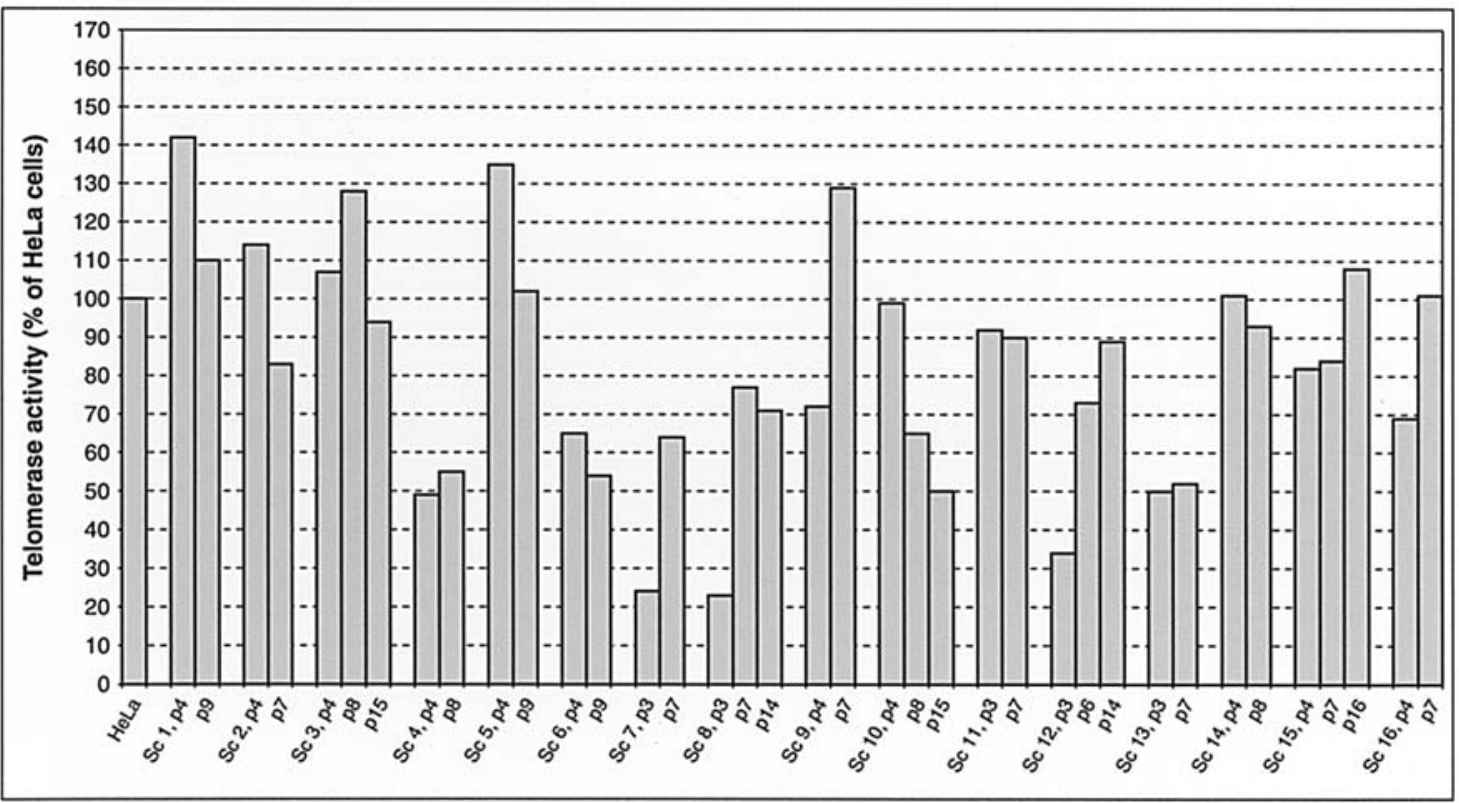

Figure 1. hTERT mRNA expression (A) and telomerase activity (B) of 16 HeLa subclones. At the indicated passages (p) following isolation, the subclones were harvested and analyzed for the levels of hTERT mRNA and telomerase activity by using real-time RT-PCR and SYBR Green real-time telomeric repeat amplification protocol (TRAP), respectively, as described in Materials and methods. The results are presented relative to the levels of hTERT expression and telomerase activity in HeLa mass culture.

were in the subclones $4,9,11$ and 13 , and the subclones 3 and 5 possessed the longest 'overlengthened' telomeres (i.e, longer telomeres than that of HeLa mass culture). Six of the 16 subclones showed a gradual increase in their telomere lengths with passage (Sc 1, 2, 3, 4, 5 and 15), while a slight decrease in telomere lengths occurred in 4 subclones (Sc 7, 8, 10 and 14) (changes shown in Fig. 2, except those of Sc 14 and 15). In the rest of the subclones (Sc 6, 9, 11, 12, 13 and 16), telomere lengths remained unchanged (shown in Fig. 2, except those of Sc 11, 12, 13 and 16). In the subclones 8 and 10 , telomeres firstly shortened, but remained unchanged in the later passages. During this process, telomeric heterogeneity of the cells significantly increased (i.e., TRF smears became larger, indicating a range of different telomeric fragment sizes within cells, as seen in Fig. 2C).

Cell morphology, telomerase expression and telomere length of HeLa subclones. Fig. 3 shows the typical cytology of the subclones with either lower levels of hTERT mRNA (Sc 9, 11 and 13) or with lower levels of both hTERT mRNA and telomerase activity (Sc 7 and 8). These clones were characterized to have more cells with a large, multinucleated 
passage

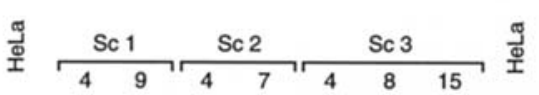

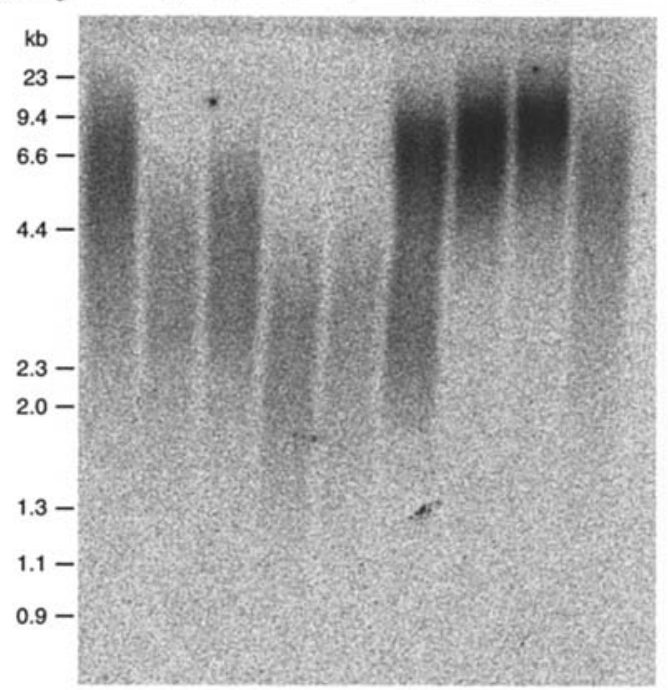

C
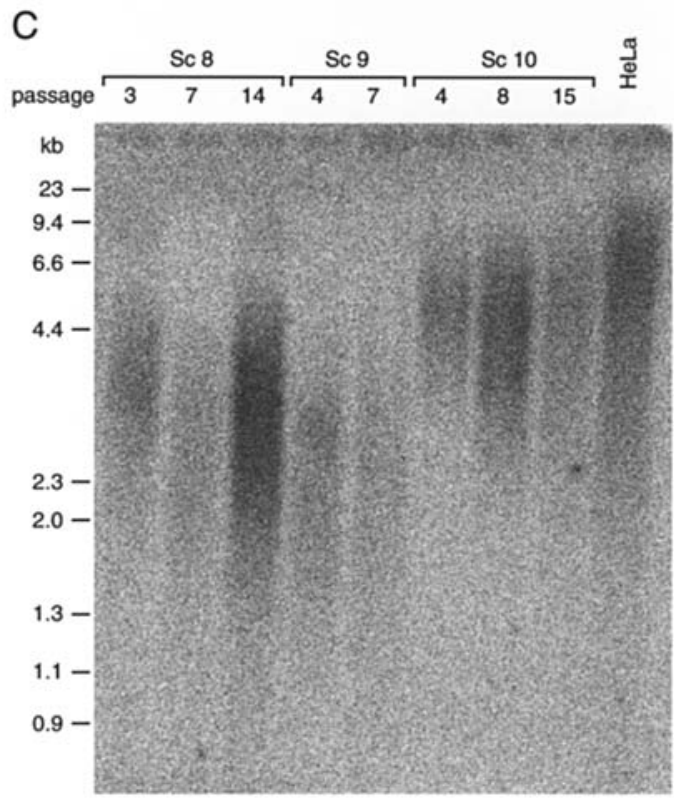

B

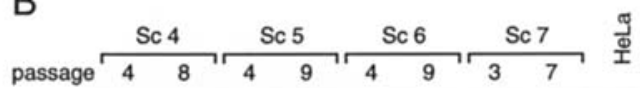

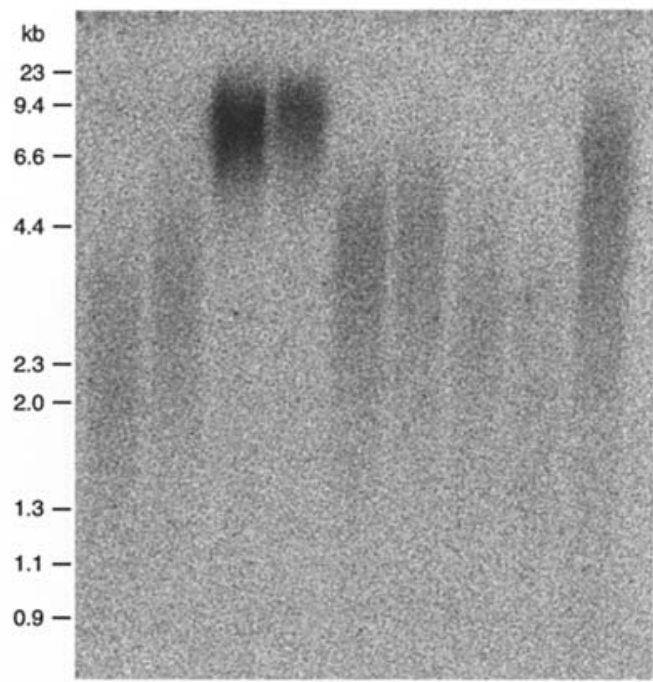

D

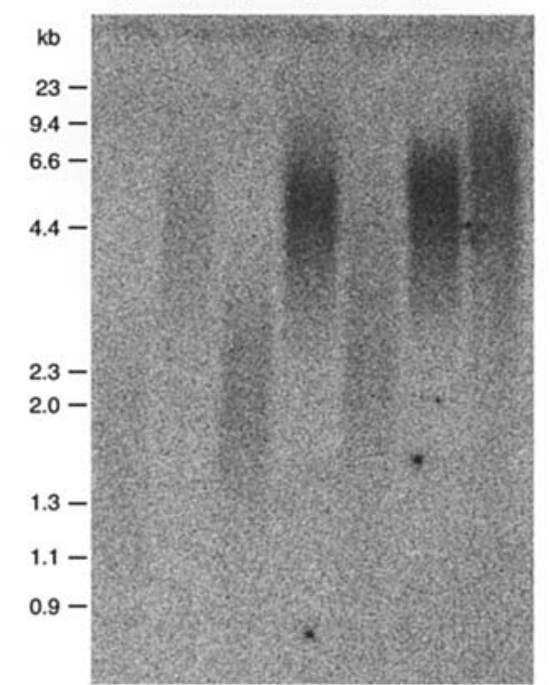

Figure 2. Telomere lengths of $16 \mathrm{HeLa}$ subclones. At the indicated passages (p) following isolation, the subclones were harvested and analyzed for the mean length of telomeric restriction fragments (TRFs) of genomic DNA by Southern blotting as described in Materials and methods. Positions of a size marker (in $\mathrm{kb})$ are given on the left.

appearance. These multinuclear cells were most prevalent in the subclones 9, 11 and 13 with short telomeres (2.0-2.3 kb). In contrast, there was only a minor fraction of cells that showed an enlarged, multinuclear phenotype in HeLa mass culture or in the subclones with higher levels of hTERT mRNA expression and telomerase activity, especially in Sc 3 and 5 (as shown in Fig. 3).

Transient transfections with siRNAs and an siRNA expression vector. Four 21-bp siRNAs targeting to different sites of the hTERT mRNA were designed as detailed in Materials and methods. The siRNA molecules were produced by a T7 transcription system in vitro. Only one of the tested siRNAs, siRNA-3, provided a potent suppression effect on hTERT expression. The siRNA-3 induced about 50\% reduction in
hTERT mRNA levels in HeLa cells at a concentration of $40 \mathrm{nM}$ $48 \mathrm{~h}$ after transfection. Telomerase activity reduction was found to correspond to the hTERT suppression (data not shown).

An expression vector encoding a hairpin siRNA directed against the effective hTERT target site was generated. The effectiveness of siRNA expressed by the vector was verified in transient transfection assays on HeLa cells. When analyzed 48 and $72 \mathrm{~h}$ post-transfection, reduction in hTERT mRNA levels was found to be similar to those seen with the transfection assays with in vitro synthesized siRNA (data not shown).

Creating long-term knockdown of hTERT. In order to achieve long-term expression of siRNA with a significant reduction in hTERT mRNA levels, we created stable transfected HeLa 

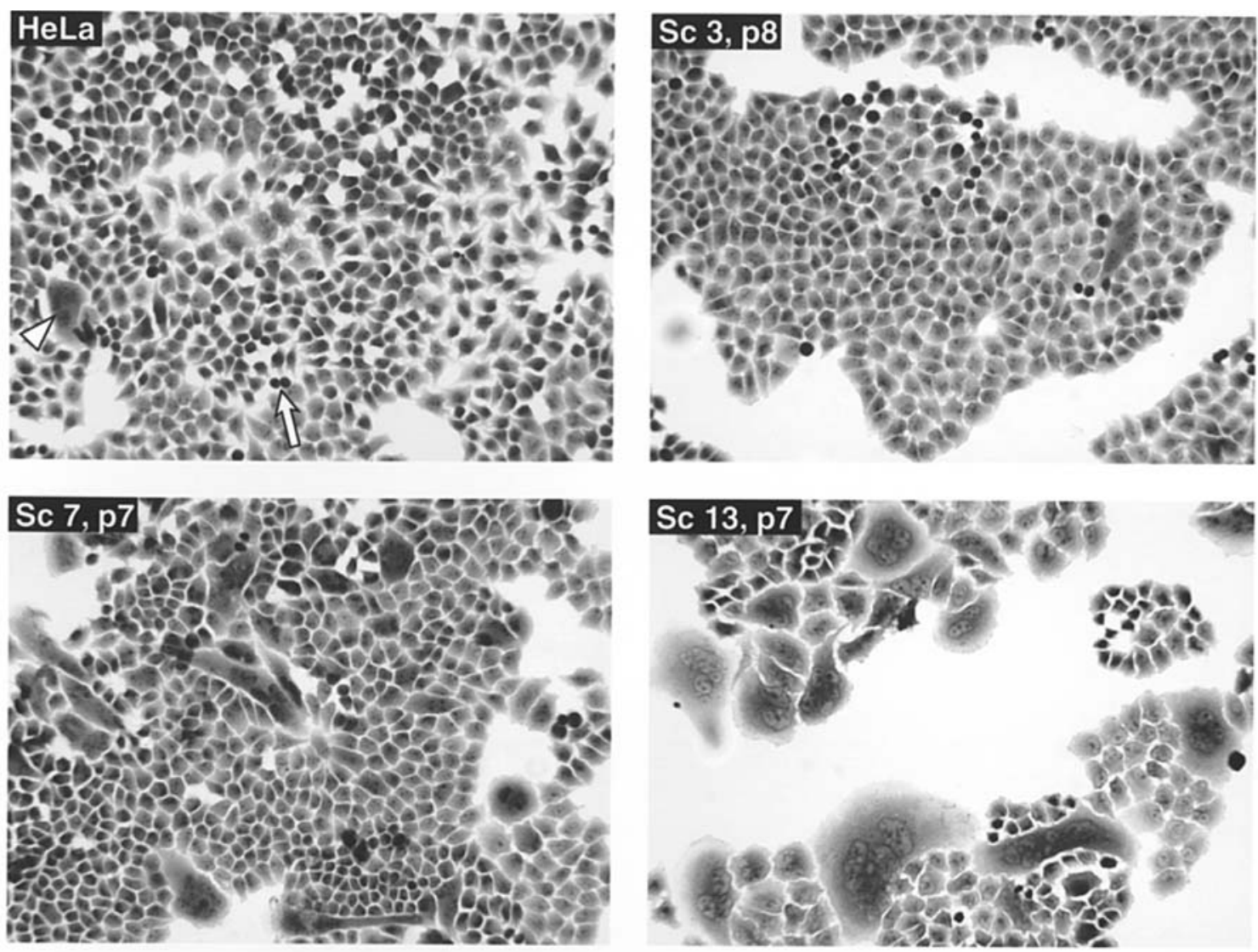

Figure 3. Cell morphology of HeLa subclones. In HeLa mass culture, the arrowhead indicates an enlarged, multinucleated cell, and the arrow indicates cells that have undergone apoptotic cell death. In HeLa and Sc 3 populations, only a minor proportion of cells exhibits an enlarged, multinuclear phenotype, while these multinuclear cells are more prevalent in Sc 7 and Sc 13 populations. All magnifications are x100.

A

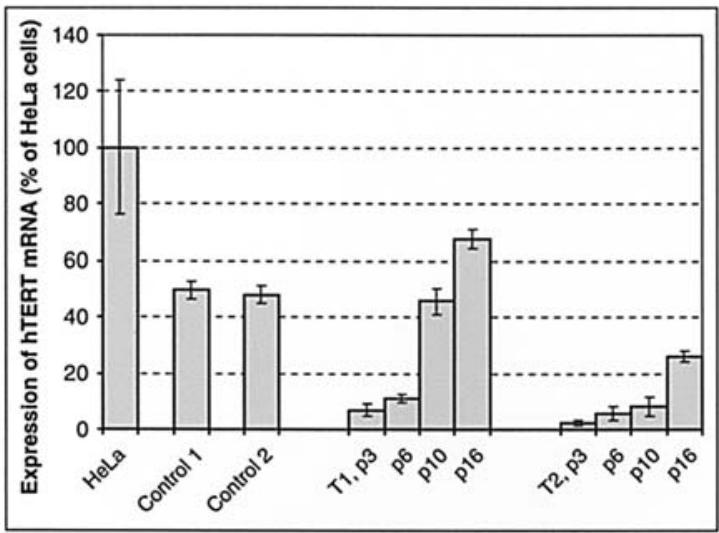

B

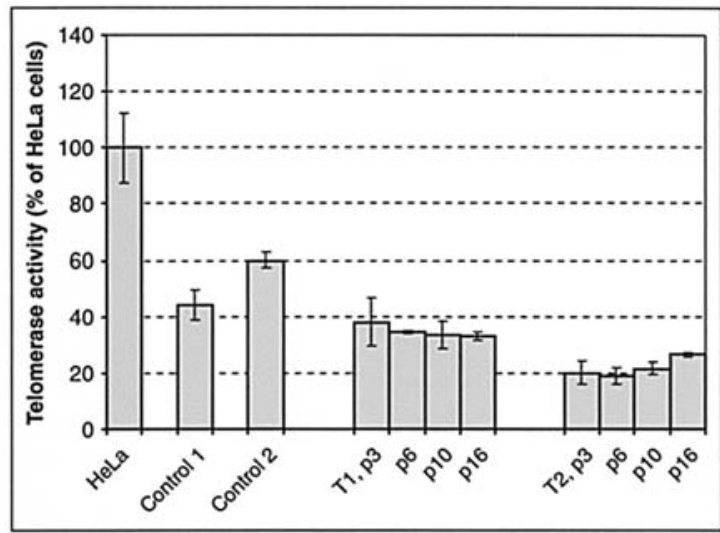

Figure 4. hTERT mRNA expression (A) and telomerase activity (B) in HeLa clones stably expressing hTERT-specific siRNA (T1 and T2). The indicated passages (p) represent time-points after transfection, as described in Materials and methods. The results are presented relative to the levels of hTERT expression and telomerase activity in HeLa mass culture. Controls are HeLa cell populations stably expressing a scrambled siRNA. The results are presented as means $\pm \mathrm{SD}$

cell clones (derived from transfected HeLa cells). After stable selection and clonal expansion, most of the 10 expanded clones showed no reduction in the expression levels of hTERT mRNA as compared to control HeLa cell populations transfected with the control siRNA vector (data not shown). However, two of the HeLa clones exhibited significantly reduced hTERT mRNA levels, and they were maintained and analyzed during long-term passaging for evaluating profiles of the clones with potent siRNA inhibitory effects on hTERT expression (clones T1 and T2; Fig. 4).

Control cell populations stably expressing a scrambled siRNA. The average hTERT mRNA level in the control cell populations (control 1 and 2) was decreased to $\sim 50 \%$ of 


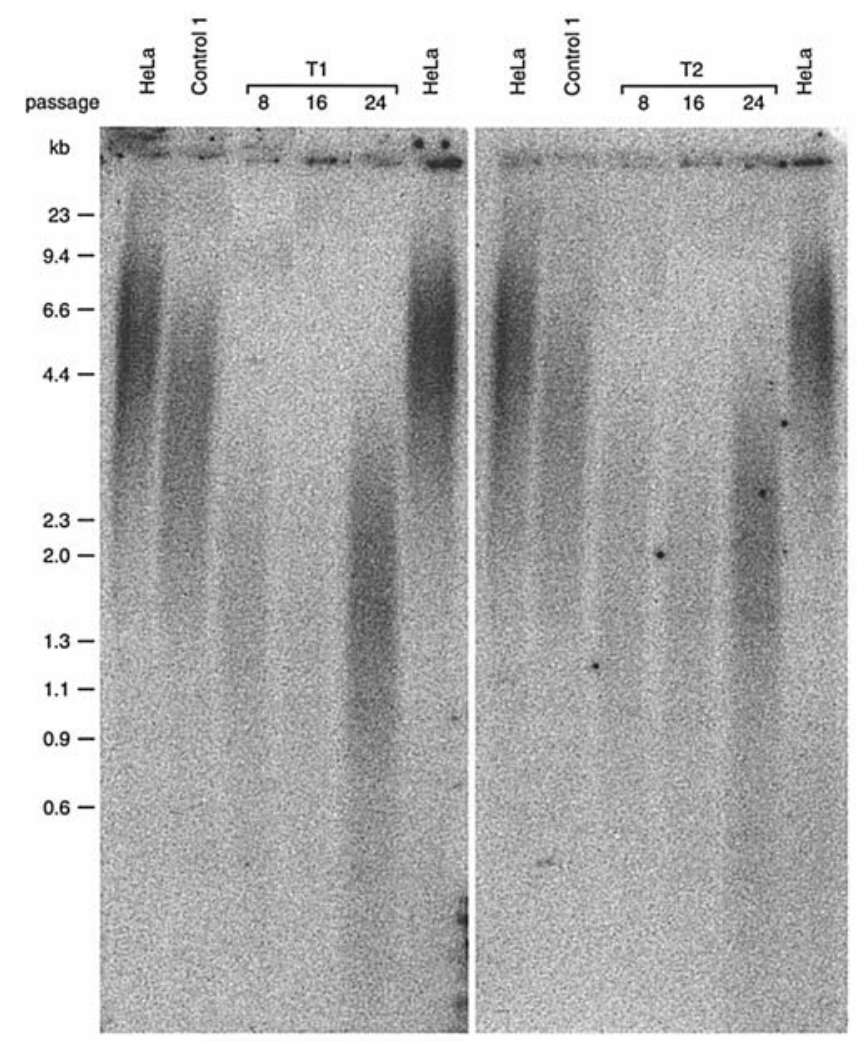

Figure 5. Telomere length analysis of HeLa clones stably expressing hTERT-specific siRNA (T1 and T2). The indicated passages represent timepoints after transfection, as described in Materials and methods. Control 1 is a HeLa cell population stably expressing a scrambled siRNA. Positions of a size marker (in $\mathrm{kb}$ ) are given on the left.

HeLa cells (Fig. 4A). Telomerase activity appeared to correlate with the remaining hTERT mRNA expression levels, being 44 and $60 \%$ of HeLa cells in the control 1 and 2, respectively (Fig. 4B). The control cell population 1 exhibited a slight decrease in its mean telomere length when compared to that of HeLa cells (Fig. 5), while telomere length of the control 2 remained unchanged (data not shown). Despite these changes, the control cell populations showed no significant changes in growth properties or cell morphology (Fig. 6).

HeLa clones stably expressing an hTERT-specific siRNA. The relative expression levels of hTERT mRNA in the clones $\mathrm{T} 1$ and $\mathrm{T} 2$ at passage 3, one month after transfection, were 7 and $2.5 \%$ of the levels of HeLa, respectively (Fig. 4A). While the clone T1 recovered hTERT expression from 11 to $46 \%$ between passages 6 to 10 , the expression level of the clone T2 remained reduced $(<10 \%)$ during passages $3-10$. The relative hTERT expression level of the clone T2 was still only $26 \%$ at passage 23 (i.e., over three months after transfection; data not shown). Fig. 4B shows that the clone $\mathrm{T} 1$ recovered telomerase-positivity, regardless of its initially low hTERT mRNA levels. In contrast, the clone $\mathrm{T} 2$ exhibited more reduced telomerase activity during a 16-passage period (relative telomerase activity 19-27\%) (Fig. 4B). Both the T1 and T2 cell populations had unusually short telomere lengths, $\sim 1.5$ and $\sim 1.7 \mathrm{~kb}$, respectively, and there was also a significantly increased telomeric heterogeneity within the cells (i.e., very large TRF smears; Fig. 5).
The growth rate of $\mathrm{T} 1$ and $\mathrm{T} 2$ cells at early passages was reduced when compared to that of the control cell populations. The inhibited cell growth was accompanied by morphological changes characteristic of senescence and/or crisis. These clones were characterized with enlarged, multinuclear cells, and in a part of these cells a vacuolated cytoplasm was a prominent feature (Fig. 6). Fig. 6 demonstrates that at passage 8, practically all detached T2 cells exhibited a senescent or apoptotic phenotype, and there existed only a minor fraction of proliferating cells. At passage 8 , the clone T1 had also a large number of cells with morphological features of both senescence and apoptosis (Fig. 6). In contrast to clone T2, there also existed a number of proliferating cells. The fraction of viable cells in clone $\mathrm{T} 1$ significantly increased at passage 12 , while clone $\mathrm{T} 2$ showed the recovery of cell viability at later passages. No significant difference in cell viability of these two clones was observed any more at passage 17 (Fig. 6).

\section{Discussion}

The present data show that there are great clonal variations in hTERT mRNA expression and telomerase activity levels within a telomerase-positive HeLa cell population. Despite the failure to show an absolute correlation between the levels of hTERT mRNA and telomerase enzymatic activity, the present findings propose that hTERT mRNA expression is one of the critical factors involved in the regulation of telomerase activity. Firstly, in 9 of the 16 subclones, a decrease or increase in hTERT mRNA expression levels was found to reflect similar changes in telomerase activity levels (Fig. 1; Sc 3, 4, 5, 7, 8, 9, 10, 12 and 15). Particularly, a significant increase in telomerase activity (1.8-3.3-fold increase in the subclones $7,8,9$ and 12) was associated also with a significant increase in hTERT mRNA levels (1.8-3.6-fold increase, respectively). Additionally, in both cases in which telomerase activity was considerably reduced (only $\sim 20 \%$ in the subclones 7 and 8 ), also hTERT mRNA levels were extremely low (<10\%) (Fig. 1). Instead, stable, unusually high hTERT expression was found to be related to high steady state levels of telomerase activity (Fig. 1; Sc 3 and 5). Consequently, hTERT mRNA expression appeared to be functionally equivalent to telomerase activity especially in cases, when hTERT was either a completely limiting factor or when hTERT was reactivated or overexpressed, thereby facilitating the assembly of active telomerase complexes. These observations strongly support the concept that hTERT has a central role in the regulation of telomerase activity. The imbalance between the levels of hTERT mRNA and telomerase activity can be, at least partly, explained by the difference in the half-lives of these factors. The half-life of hTERT mRNA is $\sim 2-3 \mathrm{~h}$ (21), and the half-life of the active telomerase complex is $\sim 24 \mathrm{~h}$ (22). Thus, the relationship between these two factors strongly depends on timing of the transcriptional upregulation or downregulation of hTERT relative to the time of collecting of the analyzed samples. Previous studies on telomerase-positive human tumor cells and cell lines have suggested that telomerase enzymatic activity is primarily regulated at the transcriptional level of both its core components, hTR and hTERT, and hTERT being the most important determinant $(23,24)$. On the other hand, study on telomerase-reconstituted fibroblast clones have provided 

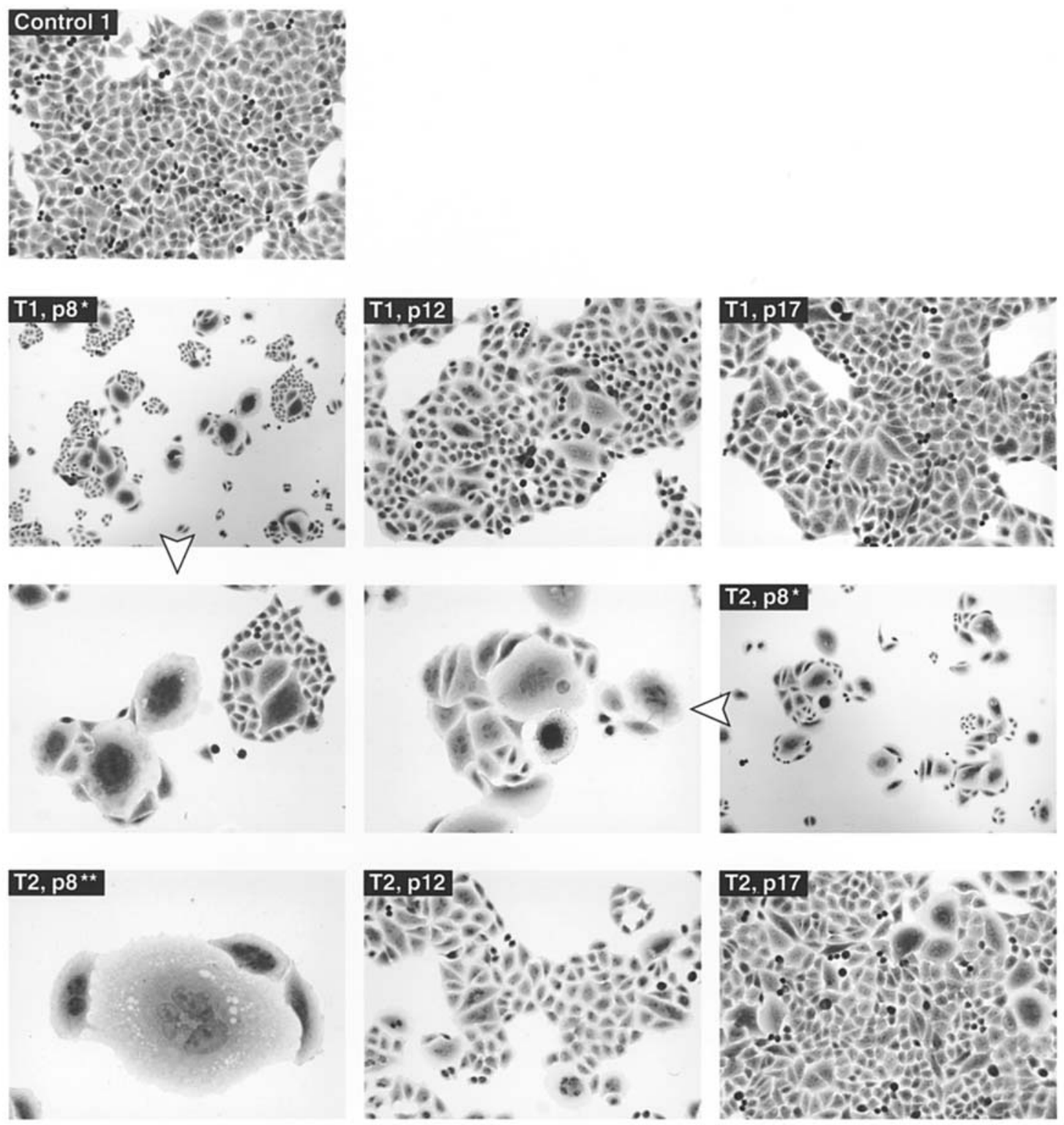

Figure 6. Cell morphology of HeLa clones stably expressing hTERT-specific siRNA (T1 and T2). Control 1 cells are HeLa cells stably expressing a scrambled siRNA. Morphological signs characteristic of senescence and/or crisis are the appearance of large, flattened multinucleated and vacuolated cell, as seen in the figure marked with $\mathrm{T} 2, \mathrm{p} 8{ }^{* *}$. Clones $\mathrm{T} 1$ and $\mathrm{T} 2$ at passage 8 are characterized with greatly enlarged, multinuclear cells reminiscent of senescence and with cells undergoing apoptotic cell death. A special feature is that cells are growing focally, indicating the exhaustion of replicative capacity. Typically, large multinucleated cells are surrounded by small 'crescent-shaped' cells. All magnifications are x100, except those marked with one asterisk are magnification $\mathrm{x} 40$ and that marked with two asterisks is magnification $\mathrm{x} 250$.

contradictory evidence (25). The expression of telomerase activity is regulated at different levels, including in addition to transcriptional regulation, post-transcriptional and posttranslational modification of hTR and hTERT $(4,5,23)$.

HeLa subclones were also found to have great variations in their telomere lengths, while HeLa mass population itself maintained a stable mean telomere length. These findings are consistent with the previous study of telomere length dynamics by Bryan et al (17). Their study also gave the first clues that the clonal telomere length heterogeneity can be at least partly due to variations in telomerase activity levels. The present observations strongly support the central role of telomerase activity level in the regulation of telomere length, as suggested also by the previous study on telomerasereconstituted fibroblast clones (25). First, both of the subclones, Sc 3 and Sc 5, that exhibited unusually high telomerase activity levels (Fig. 1B), also possessed the longest telomeres and showed a clear increase in their telomere lengths during passaging (Fig. 2A and B). Furthermore, an overall comparison of the subclones shows that telomere lengthening (Fig. 2A and B) occurred particularly in the subclones that exhibited stably high telomerase activity (Fig. 1B; $>80 \%$ telomerase activity in Sc 1, 2, 3, 5 and 15; with one exception, Sc 4 with $\sim 50 \%$ telomerase activity). In contrast, 3 of the 4 subclones that showed a slight decrease in their telomere lengths (Fig. 2B and C), either had very low telomerase activity levels (Sc 7 and 8) or had a significant reduction in telomerase activity (Sc 10) (Fig. 1B; with an exception, Sc 14 with high telomerase activity). Accordingly, high stable telomerase activity levels were closely associated with gradual telomere lengthening and particularly with 'overlengthened' telomeres, while low telomerase activity or its dramatic reduction was associated 
with telomere shortening and increased telomeric heterogeneity within cells. The relationship between telomerase activity level and telomere length, however, was not always that straightforward. Telomere shortening was found to occur also in the subclone with high telomerase-positivity, while telomere lengthening occurred also with lower steady state telomerase activity level. These findings do not preclude the role of telomerase activity in telomere length regulation, but they rather imply the involvement of additional factors in that process. TRF1, TRF2 (telomeric repeat binding factor 1, 2) and POT1 (protection of telomeres 1) are the major telomereassociated proteins that are crucial players in the regulation of telomere length, integrity and function. Both TRF1 and TRF2 are negative regulators of telomere length, and these proteins function through interaction with a variety of other proteins, implicating the complexity of the process. The protein complex formed by TRF1 is thought to negatively regulate telomere length by preventing access of telomerase to the telomeres $(26,27)$.

Despite the clonal variations in hTERT mRNA expression, telomerase activity and telomere length, HeLa mass culture proved to maintain the steady state expression levels of hTERT and telomerase and a stable mean telomere length. In HeLa subclones, telomerase activity was found to increase especially in all of the subclones with reduced activity levels, and all the subclones regained clear telomerase-positivity over time (telomerase activity $\geq 50 \%$ of that of HeLa cells). These observations propose the existence of a feedback regulation mechanism to recover telomerase activity to a necessary level for ensuring cell viability. Accordingly, most cells in a population would undergo telomerase reactivation before their telomeres shorten to a critical length. This assumption is supported by the observations that none of the HeLa subclones exhibited a growth arrest, and that only a fraction of cells in each cell population exhibited an enlarged, flattened, multinuclear phenotype that is reminiscent of a senescent cell (28). Similar findings were presented in the study concerning telomeric heterogeneity in immortal cell populations, including HeLa cell line (17). Based on the present findings, the number of senescence-like cells in a cell population seems to be closely related to the levels of hTERT expression and telomerase activity and telomere length, the frequency being much higher when the average levels of these factors are lower.

To utilize siRNA technology to knockdown gene expression, one challenge is to find effective siRNA sequences. It has been reported that siRNA efficacy is highly dependent on a target site, because the secondary structure of target RNA might prevent siRNA hybridization (29). In our study, only one of the tested siRNA sequences turned out to provide a potent knockdown effect on hTERT, while three others exhibited only minimal or no reduction in hTERT mRNA expression. In the recent study investigating the effects of antisense oligonucleotides and siRNAs targeting different sites of the hTERT mRNA, only modest reduction in hTERT mRNA expression and telomerase activity was found, and these inhibitory effects did not persist (30). These results suggest that there is a feedback mechanism that can respond to decreased hTERT mRNA levels, and cells are able to reactivate pathways to compensate for reductions in hTERT. The study using a dominant-negative mutant of hTERT has also suggested that there exists a strong selection for recovering telomerase activity in maintaining cell viability (9). These assumptions would explain the difficulties to create HeLa clones with significant hTERT reductions. Because siRNA levels expressed from a vector and thereby knockdown effects can vary among transfected cells without single-cell cloning, cells with higher levels of hTERT expression and telomerase activity may have selective growth advantage over cells with lower levels. This may be an explanation for undetectable siRNA effects in the HeLa subclone populations that were transfected separately with the hTERT-siRNA expression vector and the control vector, and analyzed after stable selection and clonal expansion (data not shown).

To study the effects of long-term suppression of hTERT expression in cancer cells, two HeLa clones stably expressing siRNA directed against hTERT were maintained and analyzed during passaging. Control cell populations appeared to receive necessary steady state levels of hTERT and telomerase (i.e., telomerase enzymatic activity correlated with hTERT expression) to maintain well their cell viability. In contrast, clones T1 and T2 stably expressing hTERT-specific siRNA evidently showed extremely reduced hTERT mRNA expression, associated with unusually short telomeres, the inhibition of cell growth and the induction of senescence and apoptotic cell death. Thus, there was obvious loss of viability in cells lacking hTERT expression and carrying short telomeres. This was most prominent in the T2 cells that showed prolonged reductions in both hTERT expression and telomerase activity. The T1 cells were found to recover their viability at earlier passages than the $\mathrm{T} 2$ cells, in line with the recovering of hTERT expression and telomerase activity in these cell populations. It can be assumed that a subpopulation of dividing cells were able to reactivate their hTERT expression and telomerase activity before reaching crisis, and these cells eventually overtook the culture. When all the present observations done with both the HeLa subclones and with the stable transfected HeLa clones are taken into account, it can be assumed that the long-term inhibitory effects of hTERT-specific siRNA in the T1 and T2 cells caused shortening of their telomeres to a critical length, thereby inducing increased senescence and cell death.

To our knowledge, only a few successful attempts have been described to inhibit hTERT expression by a vectorbased siRNA technology (31-33). Two of these studies have introduced stable hTERT inhibition by retroviral delivery of siRNAs, one in human normal fibroblasts and another in cervical cancer cells $(31,33)$. In the latter study by Nakamura et al, stable HeLa clones lacking hTERT expression were demonstrated to exhibit significantly decreased telomerase activity and shortened telomeres, eventually leading to replicative senescence (33). As indicated in our study and in the previous studies, when using an siRNA-based strategy targeting hTERT to inhibit telomerase function, there exists a delay before cell growth arrest and/or apoptosis are induced $(15,33)$. This delay is dependent on the initial telomere lengths, i.e., the number of cell divisions required for telomeres to reach a critically short length. This may represent a serious disadvantage of this therapeutic model. The reduced hTERT expression, however, has been observed to cause also immediate antiproliferative effects on cancer cells, thereby 
decreasing their tumorigenic potential (16,33). On the basis of these observations, an siRNA-based therapeutic application targeting hTERT might be most suitable for treatment of cancers with a localized and limited disease state. Strong evidence has been provided that the suppression of hTERT expression increases the susceptibility of cancer cells to ionizing radiation and chemotherapeutic agents (33-35). Thus, an siRNA-based application directed against hTERT may offer the most significant therapeutic value in combination with other DNA damaging strategies.

Our present data clearly show that long-term suppression of telomerase expression by siRNA targeting hTERT is an attainable goal, at least in a HeLa cell model system. New means to improve the delivery of siRNAs into the cells and the introduction of stable siRNA overexpression represent steps forward to their successful therapeutic applications. Thus, one of our future objectives is to construct and evaluate a herpes simples virus (HSV) vector as a producer of siRNA.

\section{Acknowledgements}

This study was supported in part by a research grant from the Govermental Special Foundation for Turku University Central Hospital, Turku, Finland, the Foundation of Turku University and the Finnish Cancer Society. The skillful technical assistance of Mrs. Niina Wahlroos is gratefully acknowledged.

\section{References}

1. Blackburn EH: Telomere states and cell fates. Nature 408: 53-56, 2000.

2. Blackburn EH: Telomeres and telomerase: their mechanisms of action and the effects of altering their functions. FEBS Lett 579: 859-862, 2005.

3. Huffman KE, Levene SD, Tesmer VM, Shay JW and Wright WE: Telomere shortening is proportional to the size of the G-rich telomeric 3'-overhang. J Biol Chem 275: 19719-19722, 2000.

4. Dong CK, Masutomi K and Hahn WC: Telomerase: regulation, function and transformation. Crit Rev Oncol Hematol 54: 85-93, 2005.

5. Janknecht R: On the road to immortality: hTERT upregulation in cancer cells. FEBS Lett 564: 9-13, 2004.

6. Kim NW, Piatyszek MA, Prowse KR, Harley CB, West MD, Ho PL, Coviello GM, Wright WE, Weinrich SL and Shay JW: Specific association of human telomerase activity with immortal cells and cancer. Science 266: 2011-2015, 1994.

7. Wright WE, Piatyszek MA, Rainey WE, Byrd W and Shay JW: Telomerase activity in human germline and embryonic tissues and cells. Dev Genet 18: 173-179, 1996.

8. Hahn WC, Stewart SA, Brooks MW, York SG, Eaton E, Kurachi A, Beijersbergen RL, Knoll JH, Meyerson M and Weinberg RA: Inhibition of telomerase limits the growth of human cancer cells. Nat Med 5: 1164-1170, 1999.

9. Zhang X, Mar V, Zhou W, Harrington L and Robinson MO: Telomere shortening and apoptosis in telomerase-inhibited human tumor cells. Genes Dev 13: 2388-2399, 1999.

10. Saretzki G, Ludwig A, von Zglinicki T and Runnebaum IB: Ribozyme-mediated telomerase inhibition induces immediate cell loss but not telomere shortening in ovarian cancer cells. Cancer Gene Ther 8: 827-834, 2001.

11. Pascolo E, Wenz C, Lingner J, Hauel N, Priepke H, Kauffmann I, Garin-Chesa P, Rettig WJ, Damm K and Schnapp A: Mechanism of human telomerase inhibition by BIBR1532, a synthetic, nonnucleosidic drug candidate. J Biol Chem 277: 15566-15572, 2002.

12. Kraemer K, Fuessel S, Schmidt U, Kotzsch M, Schwenzer B, Wirth MP and Meye A: Antisense-mediated hTERT inhibition specifically reduces the growth of human bladder cancer cells. Clin Cancer Res 9: 3794-3800, 2003.
13. Caplen NJ: RNAi as a gene therapy approach. Expert Opin Biol Ther 3: 575-586, 2003.

14. Kosciolek BA, Kalantidis K, Tabler M and Rowley PT: Inhibition of telomerase activity in human cancer cells by RNA interference. Mol Cancer Ther 2: 209-216, 2003.

15. Shammas MA, Koley H, Batchu RB, Bertheau RC, Protopopov A, Munshi NC and Goyal RK: Telomerase inhibition by siRNA causes senescence and apoptosis in Barrett's adenocarcinoma cells: mechanism and therapeutic potential. Mol Cancer 4: 24, 2005.

16. Xia Y, Lin RX, Zheng SJ, Yang Y, Bo XC, Zhu DY and Wang SQ: Effective siRNA targets screening for human telomerase reverse transcriptase. World J Gastroenterol 11: 2497-2501, 2005

17. Bryan TM, Englezou A, Dunham MA and Reddel RR: Telomere length dynamics in telomerase-positive immortal human cell populations. Exp Cell Res 239: 370-378, 1998.

18. Wege H, Chui MS, Le HT, Tran JM and Zern MA: SYBR Green real-time telomeric repeat amplification protocol for the rapid quantification of telomerase activity. Nucleic Acids Res 31: e3, 2003

19. Miller SA, Dykes DD and Polesky HF: A simple salting out procedure for extracting DNA from human nucleated cells. Nucleic Acids Res 16: 1215, 1988.

20. Elbashir SM, Harborth J, Weber K and Tuschl T: Analysis of gene function in somatic mammalian cells using small interfering RNAs. Methods 26: 199-213, 2002.

21. Xu D, Gruber A, Björkholm M, Peterson C and Pisa P: Suppression of telomerase reverse transcriptase (hTERT) expression in differentiated HL-60 cells: regulatory mechanisms. Br J Cancer 80: 1156-1161, 1999.

22. Holt SE, Aisner DL, Shay JW and Wright WE: Lack of cell cycle regulation of telomerase activity in human cells. Proc Natl Acad Sci USA 94: 10687-10692, 1997.

23. Yi X, Shay JW and Wright WE: Quantitation of telomerase components and hTERT mRNA splicing patterns in immortal human cells. Nucleic Acids Res 29: 4818-4825, 2001.

24. Saretzki G, Petersen S, Petersen I, Kölble K and von Zglinicki T: hTERT gene dosage correlates with telomerase activity in human lung cancer cell lines. Cancer Lett 176: 81-91, 2002.

25. Swiggers SJJ, Nibbeling HAJ, Zeilemaker A, Kuijpers MA, Mattern KA and Zijlmans JMJM: Telomerase activity level, but not hTERT mRNA and hTR level, regulates telomere length in telomerase-reconstituted primary fibroblasts. Exp Cell Res 297: 434-443, 2004.

26. Smogorzewska A, van Steensel B, Bianchi A, Oelmann S, Schaefer MR, Schnapp G and de Lange T: Control of human telomere length by TRF1 and TRF2. Mol Cell Biol 20: 1659-1668, 2000.

27. Rodier F, Kim SH, Nijjar T, Yaswen P and Campisi J: Cancer and aging: the importance of telomeres in genome maintenance. Int J Biochem Cell Biol 37: 977-990, 2005.

28. Yaswen P and Stampfer MR: Molecular changes accompanying senescence and immortalization of cultured human mammary epithelial cells. Int J Biochem Cell Biol 34: 1382-1394, 2002.

29. Scherr M, Morgan MA and Eder M: Gene silencing mediated by small interfering RNAs in mammalian cells. Curr Med Chem 10: 245-256, 2003.

30. Natarajan S, Chen Z, Wancewicz EV, Monia BP and Corey DR: Telomerase reverse transcriptase (hTERT) mRNA and telomerase RNA (hTR) as targets for downregulation of telomerase activity. Oligonucleotides 14: 263-273, 2004.

31. Masutomi K, Yu EY, Khurts S, Ben-Porath I, Currier JL, Metz GB, Brooks MW, Kaneko S, Murakami S, De Caprio JA, Weinberg RA, Stewart SA and Hahn WC: Telomerase maintains telomere structure in normal human cells. Cell 114: 241-253, 2003.

32. Guo Y, Liu J, Li YH, Song TB, Wu J, Zheng CX and Xue CF: Effect of vector-expressed shRNAs on hTERT expression. World J Gastroenterol 11: 2912-2915, 2005.

33. Nakamura M, Masutomi K, Kyo S, Hashimoto M, Maida Y, Kanaya T, Tanaka M, Hahn WC and Inoue M: Efficient inhibition of human telomerase reverse transcriptase expression by RNA interference sensitizes cancer cells to ionizing radiation and chemotherapy. Hum Gene Ther 16: 859-868, 2005.

34. Ludwig A, Saretzki G, Holm PS, Tiemann F, Lorenz M, Emrich T, Harley CB and von Zglinicki T: Ribozyme cleavage of telomerase mRNA sensitizes breast epithelial cells to inhibitors of topoisomerase. Cancer Res 61: 3053-3061, 2001.

35. Yuan $Z$ and Mei HD: Inhibition of telomerase activity with hTERT antisense increases the effect of CDDP-induced apoptosis in myeloid leukemia. Hematol J 3: 201-205, 2002. 